\title{
Towards the Generation of Setup Matrices from Route Sheets and Feedback Data with Data Analytics
}

\author{
Moritz Schröter ${ }^{1}$ [0000-0002-3405-9815], Ben Lütkehoff ${ }^{1}$, Markus Fischer ${ }^{1}$, \\ Matthias Blum ${ }^{1}$ and Volker Stich ${ }^{1}$ \\ ${ }^{1}$ Institute for Industrial Management (FIR) at RWTH Aachen University, \\ Campus-Boulevard 55, 52074 Aachen, Germany \\ moritz.schroeterdfir.rwth-aachen.de
}

\begin{abstract}
The function or department of production control in manufacturing companies deals with short-term scheduling of orders and the management of deviations during order execution. Depending on the equipment and characteristics of orders, sequence dependent setup times might occur. In these cases for companies that focus on high utilization of their assets due to long phases of ramp up and high energy costs, it might be optimal to choose sequences with minimal setup time times between orders. Identifying such sequences requires detailed and correct information regarding the specific setup times. With increasing product variety and shorter lot sizes, it becomes more difficult and rather time intense to determine these values manually. One approach is to analyse the relevant features of the orders described in the route sheets or recipes to find similarities in materials and required tools. This paper presents a methodology, which supports setup optimized sequencing for sequence dependent setup times through constructing the setup matrix from such route sheets with the use of data analytics.
\end{abstract}

Keywords: Production Control, Setup Time Optimizing Sequencing, Feedback Data

\section{Introduction}

In manufacturing industry, the adherence to delivery dates is one of the main logistics goals to stay competitive in a global market. Companies that use energy-intense assets such as the plastics industry also pursue a high utilization of their assets to reduce production costs per part. The following paper deals with a practical use-case derived from an ongoing research project. The overall project aims to improve production control through application of Artificial Intelligence. The specific company manufactures plastic films and bags with help of blow film extrusion in a high variety. Due to the various fields of application for plastic bags, these are available in a wide range of colors, thickness and compositions of material. Depending on several product features, the changeover processes varies depending on warm-up and cleaning phases of the machines as well as on material changeovers that require flushing and batch number restrictions. In consequence, the setup times vary between orders and currently are taken into account manually based on experience but not with different setup times in the planning system. 
Over the past years, the company has created more than 1,000 customer specific recipes stored in route sheets in the ERP-System. At this time, there is no option implemented, that visualizes known or undiscovered similarities between orders to simplify this planning step, although the production planners assume, that setup times actually vary between orders. The idea is to support a sequence dependent sequencing by calculating the setup times between orders from feedback data and building up a setup matrix. This information is a feasible input for scheduling software. The developed concept will allow companies with sequence dependent setup times to derive setup matrices automatically from once defined input data.

The remainder of this paper is organized as followed: Initially existing literature in the field of generation of setup matrices is reviewed as well as a brief description of similar problems such as paint-job sequencing. Secondly, we present our proposed methodology to define required data and the formulation of hypothesis. Finally, an extended generic setup matrix is presented to depict the results of our pursued analysis.

\section{Literature Review}

The overall goal of setup time optimizing sequencing in flow-shops and job-shops is to reduce the setup times between successive orders with sequence dependent setup times by finding a suitable, nearly optimal sequence. This requires a sufficiently large number of possible subsequent orders. Implemented correctly, such an optimization will likely reduce setup costs and at best can increase the available capacities of a workstation or even of the entire production system if the workstation is a throughput bottleneck. In the latter case, setup time optimizing sequencing can even positively affect the schedule reliability when reducing the production's backlog [1]. It should be noted, that reducing actual setup times with concepts such as SMED (Single Minute Exchange of Die) in most cases has a bigger impact on the overall optimization of production systems but cannot be realized without investments in new equipment [2].

Sequence dependent setup times occur, when different products are processed on the same machine and a change of equipment, such as tools, is necessary to manufacture a specific variant. Furthermore, the amount of time necessary must vary due to a different extent of work depending on the prior setup situation [3]. Besides tool changes, sequence dependent setup times can also occur when changing the material or additives (such as paint) when using machines that depend on a continuous flow of material like in paint shops or in our case blow film machines.

Table 1. Example for a changeover matrix with five different products

\begin{tabular}{|l|c|c|c|c|c|}
\hline From/to operation & Product 1 & Product 2 & Product 3 & Product 4 & Product 5 \\
\hline Product 1 & - & 14 & 25 & 15 & 20 \\
\hline Product 2 & 25 & - & 17 & 12 & 21 \\
\hline Product 3 & 33 & 17 & - & 18 & 10 \\
\hline Product 4 & 15 & 12 & 8 & - & 7 \\
\hline Product 5 & 20 & 25 & 35 & 5 & - \\
\hline
\end{tabular}


To depict the different setup times between variants, changeover matrices are used to consider the actual setup time between two specific product options with its features (see Table 1). Such matrices consist of discrete setup times between each product or whole product families. Similar matrices are used in paint shops with a high variety of colors to reduce flushing times between colors that otherwise would mix and result in color defects [4]. Modern production planning and scheduling tools offer the capability to consider such setup times in form of matrices in regard to specific products or product groups [5]. SAP APO even allows the automatic generation of setup groups and setup matrices from up to six characteristic values. Therefore, the individual and disjunctive characteristics must be known, which is not the case in our example [6].

In Operations Research, a vast number of heuristics exists to solve scheduling problems with sequence-dependent setup times. A comprehensive overview is presented by Allahverdi [7]. With more than 500 papers, the topic seems to be of high scientifically relevance. In contrast to this, the practice often lacks robust data to feed such algorithms. To address this issue, White and Wilson [8] develop an approach which estimates setup times by applying classification to a limited number self-recorded setup times. The classification structure consists of several elements such as the number of work pieces operated simultaneously, the loading of the work pieces and distinguishing features of products and implications to the required tools. The required data is presented with a real world example of a lathe. As a result, the setup times are estimated with a statistical regression model. Furthermore, an efficient heuristic to sequencing orders is presented. The approach does not take into account start-up phases and is limited to a set of 93 input data sets [8]. Bagherpour et al. [9] present an approach with the application of fuzzy logic to estimate setup times in sequence dependent single machine scheduling problems. In contrast to many other approaches, the setup time is not assumed as deterministic, but depends on the level of technology and the degree of the similarity of the operations carried out by the machine [9]. Unfortunately, the authors do not elaborate this statement.

Whereas in areas with discrete manufacturing such as machining, setup times mainly depend on the actual work that has to be done when doing a changeover and therefore can be related to the tools [8], in process industry the values may depend on several different factors such as the previous material color, density, batch number etc. To the best knowledge of the authors this topic hast no been discussed in research dealing explicitly with the ex-ante estimation or calculation of sequence dependent setup times from feedback data and routing tables.

\section{Deriving setup time matrices from routing tables}

The following section includes the description of the proposed methodology for generating setup matrices from route sheets and feedback data. The procedure consists of four steps (see Fig. 1), which are described in the following. The image is simplified for better comprehension; in practice, it might be necessary to do the steps 2 to 4 in an iterative manner to achieve the best results due to recognition of previously unknown features in data required. 


\begin{tabular}{|c|c|c|c|}
\hline $\begin{array}{l}\text { Process analysis of the } \\
\text { setup process }\end{array}$ & $\begin{array}{l}\text { Description of influence } \\
\text { factors on the setup time }\end{array}$ & $\begin{array}{l}\text { Analysis of gathered } \\
\text { data }\end{array}$ & $\begin{array}{l}\text { Derivation of setup } \\
\text { matrices }\end{array}$ \\
\hline $\begin{array}{l}* \text { Structured description of } \\
\text { the setup process itself } \\
\text { - Identification of order } \\
\text { induced changes } \\
* \text { Definition of desired } \\
\text { state after setup } \\
\text { activities }\end{array}$ & $\begin{array}{l}* \text { Gathering expert } \\
\text { knowledge about } \\
\text { possible influence } \\
\text { factors from interviews } \\
* \text { Definition of information } \\
\text { needed } \\
* \text { Identification of corres- } \\
\text { ponding features in data }\end{array}$ & $\begin{array}{l}* \text { Identification of feasible } \\
\text { classification algorithms } \\
\text { * Modification and } \\
\text { application of algorithms } \\
\text { * Discussion of results } \\
\text { with experts for } \\
\text { validation }\end{array}$ & $\begin{array}{l}* \text { Definition of required } \\
\text { granularity for matrix } \\
\text { columns and rows } \\
* \text { Filling of matrix with } \\
\text { calculated setup times }\end{array}$ \\
\hline
\end{tabular}

Fig. 1. Overview of the proposed methodology to generate setup matrices from route sheets and feedback data

\subsection{Process analysis of setup process}

Since the setup matrix consists of changeover times between products, the first step of the methodology includes a detailed analysis of the setup process itself. Hereby a process flow chart should give an overview of all necessary and possible activities related to the setup process. In some cases, dedicated setup plans might be available and already give a good overview of all work steps regarding the different adjustment options and changeable tools on the machine. The flow chart should not only visualize the actual order of working steps, but also include following information defined by White and Wilson [8] and be complemented by own findings:

- How the material in the machine is clamped/fixed within the machine,

- what the moving parts of the machine are and how they are adjusted (e.g. description, scale and range)

- how the material is loaded into the machine and from the machine

- which features of the loading/unloading device have to be adjusted

- what the product features are and how these are related to the tool/tools characteristics,

- what product features are influenced by the features of the former material during the run-up period of the process (e.g. colour, density, etc.).

When doing material changeovers on machines that use a continuous flow of material (such machines for film blowing machines or plastic injection molding), it has to be defined which quality of the final product can be regarded as acceptable. For applications of automated data processing, these quality features should be measurable and stored in an appropriate frequency.

\subsection{Description of known and presumed influence factors on the setup time}

The formerly described factors lead in practice to a specific setup time. In contrast to the approach of White and Wilson [8], our methodology aims at the extraction of the features from already preserved data in business applications such as the ERP-System 
(Enterprise Resource Planning) and a Process Data Acquisition (PDA). Whereas White and Wilson [8] consider manual recorded setup processes with a predefined structure, we pursue to do the regression by the analysis of the existing routing tables and orders (which include the required tools as well as the material processed in the machine.). These data is stored in different tables of the ERP-System like route sheets, past production orders and recipes. The durations for the changeover are derived from feedback data gathered at the machine from manual and automated inputs. As stated before, the changeover is seen as finished, when the product has achieved the desired quality. Therefore, we also take into account the waste produced during run-up and run-down phases. All data has been extracted by doing a so-called database dump.

Although in our case a lot of the necessary historical data is already stored in the database, the influences on the relations between several features is often only know to the production planner. In the presented case a longer cleaning run after black and red colours is necessary. Furthermore, the change of density of the final product between two processed batches is crucial to the time needed for adjustment needed during runup. With the help of expert interviews, we were able to formulate three starting hypotheses for the classification of factors. These are:

1. The changeover from a setup with for medium density films to a setup for highdensity films is less critical in terms of duration and scrap than vice versa.

2. The changeover to another colour from a red or black colour additive takes a longer run-up phase to achieve the desired colour accuracy and therefore more crap and a longer setup (ramp-up) time.

3. The changeover of the format might require a change of take-up reels and therefore a longer setup time due to more work.

Table 2. Defined input data to calculate setup times

\begin{tabular}{|l|l|l|l|}
\hline Name & format & description & source \\
\hline Order Number & string & & ERP (list of orders) \\
\hline Format & integer & & ERP (routing table) \\
\hline Colour & string & RAL Code & ERP (bill of material) \\
\hline Materials used & int & & ERP (bill of material) \\
\hline Density of final product & string & & ERP (bill of material) \\
\hline Processing time (plan) & real & time in seconds & ERP (route sheet) \\
\hline Previous order & string & & ERP (feedback data) \\
\hline Subsequent order & string & & ERP (feedback data) \\
\hline Duration of setup & real & seconds & MDA / PDA \\
\hline Duration of production run & real & seconds & MDA / PDA \\
\hline Produced scrap during run up & real & meters & MDA / PDA \\
\hline Produced scrap during run down & real & meters & MDA / PDA \\
\hline
\end{tabular}

MDA: Machine Data Acquisition; PDA: Personal Data Acquisition 
Beside the formulation of hypotheses, the required information has to be defined. The actual setup times have to be extracted from the feedback data that includes the reported time spent for setup operations. Furthermore, the weight of wasted material during the run-up phase is stored and assigned to the order. Those data can be found in a variety of business applications such as ERP-System or the Manufacturing Execution System (MES). If the data is not available, historical data can also be extracted from the machine itself, e.g. an installed process control system. In other cases, it might be even necessary to digitize handwritten or unstructured data from printed templates. Table 2 gives an overview of the defined data and data sources for our use case.

\subsection{Analysis of gathered data}

The next step in automated generation of setup matrices is the analyses of the previously defined data. The most feasible algorithms have to be defined yet. Nevertheless, we present a conceptual problem description to execute multivariable regressions.

To describe the characteristics that have an impact on the actual setup times we propose a classification scheme based on a tuple notation. In a tuple notation characteristics are referenced by a symbolic notation, so that instead of the multitude of possible order characteristics, a particular setup situation can be briefly described by a tuple [10]. For our example of the blow film machine, we identified three main characteristics.

1. Density of main ingredient $\alpha_{1} \in\{\mathrm{H}, \mathrm{L}, \mathrm{Lin}\}$ whereby $H$ is for "High Density", $L$ is for "Low Density" and Lin is for linear low-density polyethylene.

2. Desired colour of the final product $\alpha_{2} \in\{\circ, b, r\}$ whereby $\circ$ is for any colour except $r$ for red and $b$ for black.

3. Format of the uncut film $\alpha_{3} \in\{06,08,10,12,16\}$ whereby the number gives the wide of the film in decimetres.

The corresponding data will be stored in one database by joining different tables from the various IT-systems. It might be necessary to perform some prior calculations, such as the derivation of the setup time from two timestamps. This highly depends on the specific structure of the data. With help the of regression analysis such as regression trees [11] or using pivot tables, each tuple-set of an order will be compared to the tupleset of the previously processed order. From those combinations, specific values are calculated whenever possible. With an increasing number of characteristics, (exceeding the three initially defined), it might be necessary to classify orders in terms of several attributes. Those must not consist of only one characteristic, but also can represent a combination of characteristics.

\subsection{Derivation of setup matrices}

The results of the regression analysis and changeover process classification shall lead to quantitative relationships between setup characteristics. Those are depicted in an extended setup matrix (see Table 3 ). The highlighted elements mark the concept of visualizing changeover situations that have shown major deviations from the planned setup- 
times in the past. This information could result in the planning decision to schedule a utility worker for the affected shift. The table only includes production orders and not all possible combinations.

Table 3. Example for an extended setup matrix generated from historical data

\begin{tabular}{|l|c|c|c|c|c|}
\hline From/to Order & Order 715 & Order 716 & Order 717 & Order 718 & Order 719 \\
\hline \multirow{2}{*}{ Order $715(\mathrm{~L}|\circ| 10)$} & - & $\mu=13,2$ & $\mu=10,3$ & $\mu=14,7$ & $\mu=11,5$ \\
& & $\sigma=3,6$ & $\sigma=2,5$ & $\sigma=3,3$ & $\sigma=2,8$ \\
\hline \multirow{2}{*}{ Order $716(\mathrm{H}|\circ| 08)$} & $\mu=17,3$ & - & $\mu=17,6$ & $\mu=10,7$ & $\mu=14,3$ \\
& $\sigma=4,4$ & & $\sigma=4,5$ & $\sigma=2,6$ & $\sigma=3,1$ \\
\hline \multirow{2}{*}{ Order $717(\mathrm{~L}|\circ| 12)$} & $\mu=5,1$ & $\mu=13,7$ & & $\mu=9,6$ & $\mu=11,8$ \\
& $\sigma=1,2$ & $\sigma=3,4$ & - & $\sigma=1,9$ & $\sigma=2,9$ \\
\hline \multirow{2}{*}{ Order $718(\mathrm{H}|\mathrm{r}| 12)$} & $\mu=21,2$ & $\mu=13,1$ & $\mu=4,3$ & - & $\mu=22,7$ \\
& $\sigma=5,2$ & $\sigma=2,7$ & $\sigma=0,9$ & $\sigma=5,5$ \\
\hline \multirow{2}{*}{ Order $719(\mathrm{~L}|\mathrm{~b}| 08)$} & $\mu=14,1$ & $\mu=13,3$ & $\mu=15,2$ & $\mu=18,7$ & - \\
& $\sigma=2,8$ & $\sigma=2,8$ & $\sigma=3,1$ & $\sigma=3,9$ & - \\
\hline
\end{tabular}

Compared to static setup matrices, the proposed extended setup matrix combines two new aspects: Instead of relating to a specific product or material number, actual orders and the main characteristics for the changeover are visualized. These helps when dealing with a big variety of material. Since the data has some uncertainty, we state the mean value $\mu$ as well as the sample standard deviation $\sigma$ of the analyzed setup times. The setup matrix can either be used as a visual support the production planner or (reduced to its mean values) further processed for scheduling tools such SAP APO [12]. The setup matrices should not only be considered for already known setup situations, but adapt to new possible sequences. Those arise from new rout sheets, which are created when designing new products. Therefore, a continuous analysis of recipes, orders and feedback data is advised.

\section{Conclusion and Outlook}

Setup matrices are required to schedule setup time optimizing sequences to increase the utilization of costs intense assets. It has been shown, that most research work assumes deterministic setup matrices but only few deal with the determination of actual setup times. With an increasing number of product variants, the number of different route sheets and varying setup times increases as well. The paper presents an approach to generate setup matrices by analyzing routing tables and feedback data. In the next step, the formulated hypotheses will be analyzed to do the classification. The results will then be discussed with the experts from the company and adjusted where necessary. In future research it has to be evaluated which data analysis approach (e.g. data mining) and which algorithm is the most suitable for such a problem. 


\section{Acknowledgement}

The research project iProd is funded by the European Union (grant number EFRE/ERDF-0800924) in the context of the Operational Program for the promotion of investments in growth and employment for North Rhine-Westphalia from the European fund for regional development (OP ERDF NRW).

\section{References}

1. Lödding, H.: Handbook of manufacturing control. Fundamentals, description, configuration. Springer, Heidelberg (2013). doi: 10.1007/978-3-642-24458-2

2. Shingo, S.: A revolution in manufacturing. The SMED system. Productivity Press, Cambridge, Mass. (1985)

3. Allahverdi, A., Ng, C.T., Cheng, T.C.E., Kovalyov, M.Y.: A survey of scheduling problems with setup times or costs. European Journal of Operational Research (2008). doi: 10.1016/j.ejor.2006.06.060

4. Spieckermann, S., Gutenschwager, K., Voß, S.: A sequential ordering problem in automotive paint shops. International Journal of Production Research (2007). doi: 10.1080/00207540310001646821

5. Herrmann, J.W. (ed.): Handbook of Production Scheduling. International Series in Operations Research \& Management Science, vol. 89. Springer Science+Business Media Inc, Boston, MA (2006). doi: 10.1007/0-387-33117-4

6. SAP SE: Automatic Generation of Setup Groups and Setup Matrices. https://help.sap.com/viewer/c95f1 f0dcd9549628efa8d7d653da63e/7.0.4/en-US/fba1ff3f8297-4796-b1e7-e29609094230.html. Accessed 27 March 2018

7. Allahverdi, A.: The third comprehensive survey on scheduling problems with setup times/costs. European Journal of Operational Research (2015). doi: 10.1016/j.ejor.2015.04.004

8. White, C.H., Wilson, R.C.: Sequence dependent set-up times and job sequencing. International Journal of Production Research (1977). doi: 10.1080/00207547708943117

9. Bagherpour, M., Noghondarian, K., Noori, S.: Applying Fuzzy Logic to Estimate Setup Times in Sequence Dependent Single Machine Scheduling Problems. IJCSNS International Journal of Computer Science and Network Security 7(1), 111-118 (2007)

10. Graham, R.L., Lawler, E.L., Lenstra, J.K., Kan, A.H.G.R.: Optimization and Approximation in Deterministic Sequencing and Scheduling. A Survey. In: Hammer, P.L., Johnson, E.L., Korte, B.H. (eds.) Annals of Discrete Mathematics : Discrete Optimization II, vol. 5, pp. 287-326. Elsevier (1979). doi: 10.1016/S0167-5060(08)70356-X

11. Rokach, L.: Data mining with decision trees. Theory and applications, 2nd edn. Series in Machine Perception and Artificial Intelligence, vol. 81. World Scientific Pub. Co, Singapore, Hackensack, N.J (2015)

12. SAP SE: Setup Matrix for Characteristic Values. https://help.sap.com/viewer/c95f1 f0dcd9549628efa8d7d653da63e/7.0.4/en-US/d7dc54d73c9f-413f-ae15-83ae04b948d3.html (2018). Accessed 27 March 2018 